\title{
Did aging affect the electromechanical delay in Karate practitioners during Mae-Geri kick performance?
}

\author{
Mário A. RODRIGUES-FERREIRA*1,2,3, António M. VENCESBRIT01,2,3,4, \& Marco A. C. BRANCO ${ }^{1}$ \\ ${ }_{1}^{1}$ Sports Sciences School of Rio Maior, Polytechnic Institute of Santarém - ESDRM-IPS (Portugal) \\ ${ }^{2}$ Life Quality Research Centre - CIEQV (Portugal) \\ ${ }^{3}$ Research Unit of the Polytechnic Institute of Santarém - UIIPS (Portugal) \\ ${ }^{4}$ International Martial Arts and Combat Sports Scientific Society - IMACSSS (Poland)
}

\section{Introduction}

Electromechanical delay (EMD) is defined as the time interval between the onset of the electrical activity in a muscle and the beginning of movement, that is, the time lag between muscle activation and muscle force production (Cavanagh \& Komi, 1979), and represents the motor units' activation and shortening of the series elastic component of the musculoskeletal system (Grabiner, 1986). It has been reported that EMD increase with aging, between 18 and 60, using supramaximal stimulation (Yavuz, et al., 2010). Although there are no studies that analyze the effects of aging on EMD during sport specific motor skills, especially in karate. Therefore, the aim of the study was to analyze the effects of aging on EMD in karate practitioners during the performance of the mae-geri kick.

\section{Methodology}

The sample was composed by 21 young male karate practitioners (YgK) and 16 non-karate practitioners (NK), aged between 18 and 35 years old, and 9 veteran karate practitioners, aged between 50 and 63 years old (VetK). All participants were volunteered to participate in the study and signed an informed consent document. The motor skill consisted in the performance of the mae-geri kick, with the right lower limb (dominant), starting from the karate typical stance of zenkutsu dachi, and ended when the foot hits the target area of the training bag with the lower part of the metatarsals - koshi. The participants performed three fast and strong repetitions of the maegeri kick, with a rest period of 30 seconds between repetitions. The median value of the three trials was used for analysis. The kick movement was filmed with a high-speed camera (Casio EX-FH20) and the kinematic analysis was held with the software Ariel Performance Analysis System (APAS, Ariel Dynamics-2003). Surface electromyographic (EMG) activity was recorded from rectus femoris (RF) and vastus lateralis (VL) portions of the quadriceps femoris, long head of the biceps femoris (BF), tibialis anterior (TA) and external portion of the gastrocnemius (GA). Statistical analysis was performed with the SPSS (version 17.0), with statistical significance set at $p<0.05$. Normality (Shapiro-Wilks test) and homogeneity (Levene's test) were satisfied for a significance level of .05. The One-Way Analysis of Variance (ANOVA) was used to analyze the differences between groups, with the Tukey HSD Post-Hoc.

\section{Results}

In the pelvic girdle movement, NK presented a significantly higher EMD (msec.) than YgK in the BF (45.8 vs. -86.7; $p=0.001$ ) and RF ( 8.4 vs. $59.1 ; p=0.027$ ). In the hip flexion movement, YgK presented

*Email: marioarferreira@esdrm.ipsantarem.pt 
a significantly shorter EMD in the RF than NK (32.3 vs. 103.7; $p=0.036)$ and VetK (32.3 vs. 127.4; $p=0.015)$, and in the BF than NK (-64.6 vs. 85.1; $p=0.001)$. In the knee extension movement, $\operatorname{YgK}$ presented a significantly shorter EMD in the RF than NK (133.7 vs. 198.3; $p=0.019)$ and VetK (133.7 vs. 204.9; $p=0.029$ ), and in BF than NK (38.5 vs. 179.8; $p=0.001)$.

\section{Discussion and conclusion}

It was observed that YgK presented a smaller EMD than NK in the agonist and antagonist muscles of hip flexion and knee extension movements suggesting smaller time needed for motor unit activation and shortening of the musculoskeletal system (Grabiner, 1986) during the performance of the mae-geri kick, and those results might be related with specific training of karate. VetK presented a significantly higher EMD than YgK in the RF during the hip flexion and knee extension, and those differences seem to be related with aging (Yavuz, et al., 2010), who could promote a decrease in tendon stiffness (Karamanidis \& Arampatzis, 2005), the deterioration of tendon material (Magnusson, et al., 2008) and the loss of muscle fibers and contractile capacity despite the fact that VetK remain physically active (5.3 hours per week; 35 years of practice). The study was limited by the fact that the iliopsoas muscle, main agonist of the hip flexion, was not studied.

In conclusion, the karate practice seems to reduce the electromechanical delay but the VetK presented a prolonged EMD in the agonist muscle responsible for the beginning of the mae-geri kick movement, suggesting that aging has a negative impact on the contractile capacity of the muscles.

\section{References}

Cavanagh, P. R., \& Komi, P. V. (1979). Electromechanical delay in human skeletal muscle under concentric and eccentric contractions. European Journal of Applied Physiology, 42(3), 159-63.

Grabiner, M. D. (1986). Bioelectric characteristics of electromechanical delay preceding concentric contraction. Medicine and Science in Sports and Exercise, 18(1), 37-43.

Karamanidis, K., \& Arampatzis, A. (2005). Mechanical and morphological properties of different muscle-tendon units in the lower extremity and running mechanics: effect of aging and physical activity. The Journal of Experimental Biology, 208(20), 3907-23.

Magnusson, S. P., Narici, M. V., Maganaris, C. N., \& Kjaer, M. (2008). Human tendon behaviour and adaptation, in vivo. The Journal of Physiology, 586(1), 71-81.

Yavuz, S. U., Sendemir-Ürkmez, A., \& Türker, K. S. (2010). Effect of gender, age, fatigue and contraction level on electromechanical delay. Clinical Neurophysiology, 121(10), 1700-6.

Key words: Electromyography; motor control; combat sports; martial arts; karate. 